\title{
Pure red cell aplasia associated with thymoma: a report of a single-center experience
}

\author{
Satoru Moriyama ${ }^{1}$, Motoki Yano ${ }^{2}$, Hiroshi Haneda ${ }^{1}$, Katsuhiro Okuda ${ }^{1}$, Osamu Kawano ${ }^{1}$, Tadashi \\ Sakane $^{1}$, Risa Oda ${ }^{1}$, Takuya Watanabe ${ }^{1}$, Ryoichi Nakanishi ${ }^{1}$ \\ ${ }^{1}$ Department of Oncology, Immunology and Surgery, Nagoya City University Graduate School of Medical Sciences, Nagoya, Japan; ${ }^{2}$ Division of \\ Chest Surgery, Department of Surgery, Aichi Medical University, Nagakute, Japan \\ Contributions: (I) Conception and design: S Moriyama, M Yano; (II) Administrative support: R Nakanishi; (III) Provision of study materials or \\ patients: S Moriyama, H Haneda, K Okuda, O Kawano; (IV) Collection and assembly of data: S Moriyama, T Sakane, R Oda, T Watanabe; (V) Data \\ analysis and interpretation: S Moriyama, M Yano; (VI) Manuscript writing: All authors; (VII) Final approval of manuscript: All authors. \\ Correspondence to: Motoki Yano. Division of Chest Surgery, Department of Surgery, Aichi Medical University, 1-1 Yazakokarimata, Nagakute 480- \\ 1195, Japan. Email: yano.motoki.369@aichi-med-u.ac.jp.
}

Background: Pure red cell aplasia (PRCA) associated with thymoma is relatively rare, and relevant reports are limited. We investigated the clinical features and outcomes of PRCA associated with thymoma in this study.

Methods: A retrospective review of all PRCA patients who underwent surgical resection of thymoma from April 1, 2004, to December 31, 2015, was performed.

Results: We experienced eight patients with PRCA among 146 patients who underwent surgical resection of thymoma. Extended thymectomy $(n=4)$ and thymectomy $(n=4)$ were performed for thymoma. Regarding the WHO classification of thymoma, the subtypes were type B2 or B3 in seven patients, and the stage of thymoma was advanced in seven patients. Complete resection was achieved macroscopically in only five patients. Recurrence of thymoma occurred in four patients who underwent complete resection. PRCA was diagnosed after surgical resection of thymoma in six patients (range 1-101 months, median 56.5 months). Cyclosporine was used for PRCA in six patients. Pneumonia of treatment-related complications due to cyclosporine occurred in all patients. The follow-up period ranged from 13-147 months (median 54.5 months) after the PRCA diagnosis. Three patients obtained complete remission of anemia by cyclosporine. Although one patient was able to stop taking cyclosporine because of complete remission of anemia, transfusion was needed due to relapse of PRCA. Five patients died, with the main causes of death diagnosed as pneumonia $(n=4)$ and cardiac failure $(n=1)$.

Conclusions: PRCA associated with thymoma was diagnosed postoperatively in three-quarter of patients. We should be alert for the occurrence of PRCA even after resection of thymoma, especially in patients with incomplete resection or advanced disease. Cyclosporine was effective for PRCA, but treatment-related complications occurred, particularly pneumonia. As treatment for PRCA associated with thymoma and its complications were combined in a complex manner, treating PRCA associated with thymoma can be quite difficult.

Keywords: Pure red cell aplasia (PRCA); thymoma; cyclosporine

Submitted Feb 08, 2018. Accepted for publication Jun 28, 2018.

doi: $10.21037 /$ jtd.2018.07.14

View this article at: http://dx.doi.org/10.21037/jtd.2018.07.14 


\section{Introduction}

The first case of pure red cell aplasia (PRCA) associated with thymoma was reported in 1930 by Polayes and Lederer (1). PRCA patients were associated with thymoma in approximately $8.5-50 \%$ of all cases (2-4), and approximately $2-5 \%$ of thymoma patients developed PRCA $(2,5)$. Acquired pure red cell aplasia associated with thymoma is relatively rare, and relevant reports are limited. Recently, the longterm response and outcomes following immunosuppression have been reported in patients with idiopathic, thymomaassociated, or large granular lymphocyte leukemiaassociated PRCA, and risk factors for death in patients with these disorders were identified (6).

In the present study, we investigated the clinical features and outcomes of patients with PRCA associated with thymoma.

\section{Methods}

A retrospective review of all PRCA patients who underwent surgical resection of thymoma at Nagoya City University Hospital from April 1, 2004, to December 31, 2015 , was performed. Patients' clinical data, including their characteristics, pathology reports, treatment regimens, complications, and outcomes, were extracted from the medical records. The histological classification of the thymoma was based on criteria of WHO classification (7). Clinical pathological staging of thymoma was performed according to the criteria proposed by Masaoka et al. (8). The diagnosis of PRCA was based on the criteria proposed by Dessypris and Lipton (9). Erythrocytes are normochromic and normocytic on peripheral blood counts. Reticulocytes account for $<1 \%$ of counts, and erythroblasts are either totally absent or constitute less than $1 \%$ of the marrow differential counts. Possible causes of acquired PRCA, including the patients' prescription history, preceding infections and immunological examination results, were explored. Complete remission for PRCA was defined as maintaining hemoglobin levels at $>11.0 \mathrm{~g} / \mathrm{dL}$ without transfusion. This study was approved by the Institutional Review Boards of Nagoya City University Hospital (No. 70-00-0038) and carried out in accordance with the Declaration of Helsinki. All of the patients consented to the use of their tissues for the present analysis.

\section{Results}

We experienced eight patients with PRCA among 146 patients who underwent surgical resection of thymoma at Nagoya City University Hospital from April 1, 2004, to December 31, 2015. There were five males and three females, with a mean age at the surgical resection of 59 (range 45-80 years old). The subtypes of thymoma were A $(n=1), B 2(n=5)$, and B3 $(n=2)$ according to the WHO classification, and the stages were II $(\mathrm{n}=1)$, III $(\mathrm{n}=3), \mathrm{IVa}$ $(\mathrm{n}=2)$, and $\mathrm{IVb}(\mathrm{n}=2)$ according to Masaoka's classification. One patient (No. 4) had a complication of myasthenia gravis and idiopathic thrombocytopenic purpura (Table 1). Myasthenia gravis was diagnosed before surgical resection of thymoma, and idiopathic thrombocytopenic purpure was diagnosed at 133 months after surgical resection.

Five patients received preoperative chemotherapy using cytotoxic agents $(n=1)$ and high-dose steroids $(n=4)$. Extended thymectomy $(n=4)$ and thymectomy $(n=4)$ were performed in eight patients with thymoma. Complete resection was achieved macroscopically in only five patients. Postoperative radiotherapy was given to five patients. One patient (No. 3) received adjuvant hemithoracic radiation (15 Gy). Four patients were treated with postoperative mediastinal radiation to the tumor bed (40-56 Gy). A recurrence of thymoma occurred in four patients who underwent complete resection (Table 1).

The diagnosis of PRCA was made based on the results of bone marrow biopsies and peripheral blood counts. Six patients were diagnosed with PRCA after surgical resection of thymoma (range 1-101 months, median 56.5 months), and two patients were diagnosed before surgical resection (Table 1, Figure 1). Neither of the two patients diagnosed with PRCA before operation achieved complete remission of anemia after surgical resection of thymoma. Cyclosporine was used to treat PRCA in six patients with or without corticosteroids, and immunosuppressive agents were not used in the other two patients, who only received occasional transfusion. Three patients (including two who died) obtained complete remission of anemia by cyclosporine with or without corticosteroids. The patient who was still alive had continued to take cyclosporine. In one patient (No. 6), cyclosporine was stopped after 12 months because of complete remission of anemia. However, transfusion was needed after 72 months' interruption due to the relapse of 


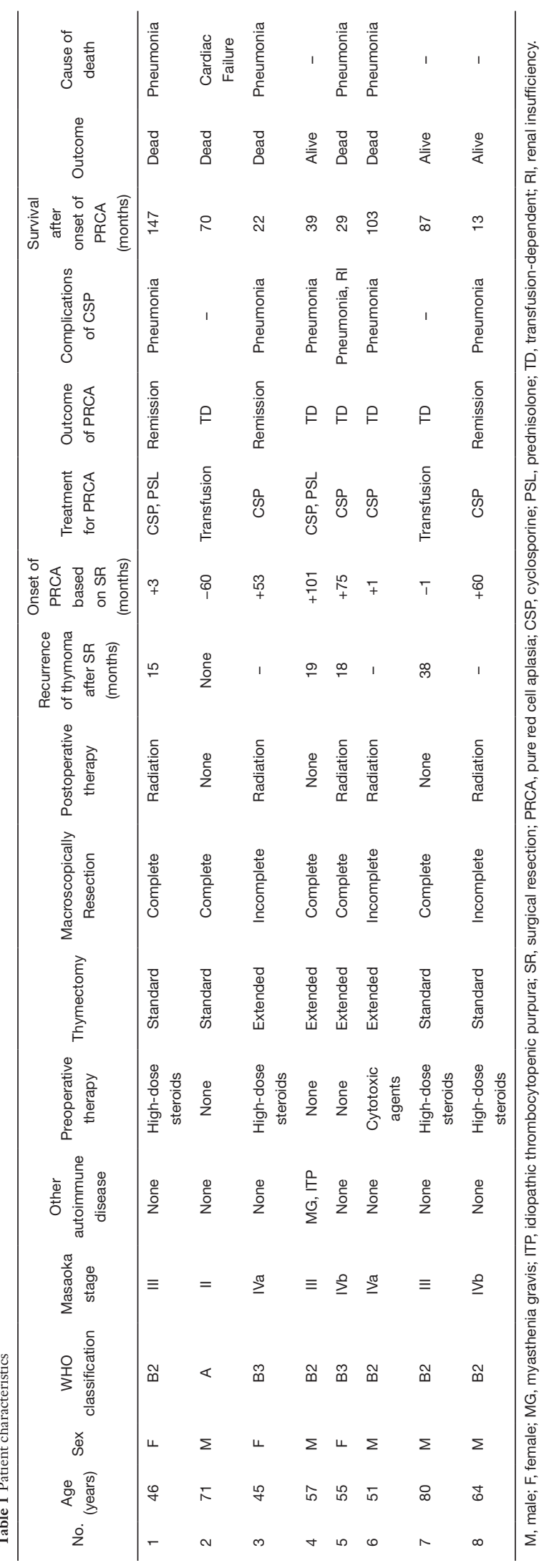

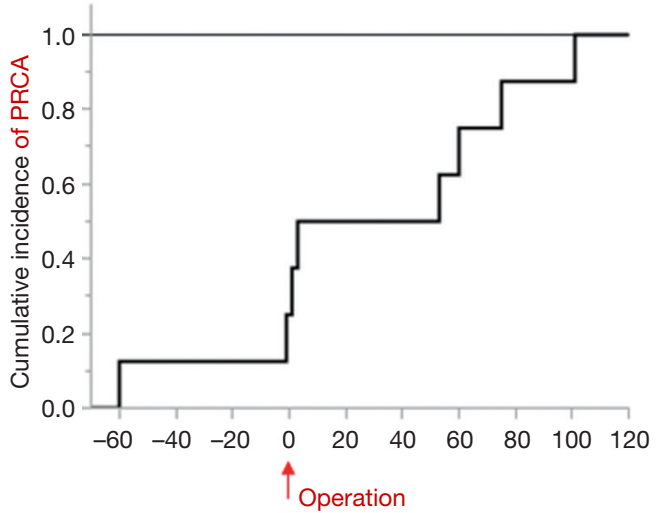

Time from surgical resection to the occurrence of PRCA (months)

Figure 1 The chronological arrangement of surgical resection for thymoma and the occurrence of PRCA. The time at which each patient underwent surgical resection was set at point 0 on the $\mathrm{x}$-axis (arrow). Six patients were diagnosed with PRCA after undergoing surgical resection of thymoma, and two were diagnosed before undergoing surgical resection.

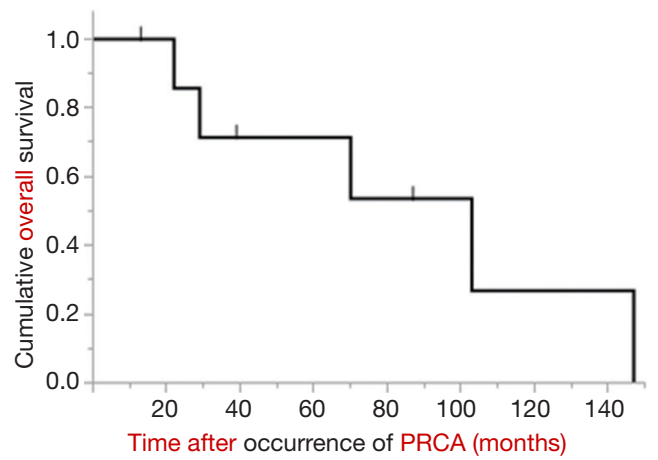

Figure 2 The overall survival of all PRCA patients with thymoma. PRCA, pure red cell aplasia.

PRCA (Table 1).

As treatment-related complications of cyclosporine, pneumonia was seen in all patients, and renal insufficiency was observed in one of the six patients who received the agent. Pneumonia was seen in all patients who received postoperative radiotherapy (Table 1). The follow-up period ranged from 13-147 months (median 54.5 months) after the PRCA diagnosis (Figure 2). Five patients ultimately died, with the main causes of death being pneumonia $(n=4)$ and cardiac failure $(\mathrm{n}=1)$.

\section{Discussion}

Our study confirmed the clinical features and outcomes 
in patients with PRCA associated with thymoma. Table 2 summarizes an overview of similar cases reported in the literature. In this study, the histology of thymoma was mainly type B2 and B3, and the pathological stage was primarily advanced disease. The thymoma histology has varied in some reports $(10,11,13)$ but was mainly type A or $A B$ in early reports (14). The pathological stage in our study was consistent with those noted in later reports (12) but contrasted with those in early reports (14). These different findings may have been influenced by the rarity of PRCA associated with thymoma.

Surgical resection of the thymoma associated with PRCA has been recommended as the initial treatment because it leads to remission in $25-38 \%$ PRCA patients $(14,15)$. In a previous study, two patients achieved complete remission of PRCA after extended thymectomy (12). In our study, PRCA associated with thymoma was diagnosed postoperatively in six of eight patients, and the remaining two diagnosed with PRCA before the operation failed to obtain complete remission of anemia after surgical resection of thymoma. Surgical resection of thymoma was performed in 36 patients in a previous study, 16 of whom were diagnosed with PRCA post-thymectomy (10). In another study, none of the 12 patients who underwent surgery obtained complete remission of anemia after surgical resection alone (11). The role of thymoma and the significance of thymectomy in the pathogenesis of anemia remain unclear. Masuda et al. reported that a patient with PRCA associated with thymoma had clonal T-cell expansions in the thymoma and peripheral blood (16). However, Fujishima et al. reported that a patient with PRCA associated with thymoma had clonal T-cell expansions in only the peripheral blood, not in the thymoma (17). The significance of thymoma with PRCA in providing conditions for the clonal expansions of pathogenic $\mathrm{T}$ cells may differ among patients (10).

Cyclosporine induced an effective response in $95 \%$ patients with PRCA associated with thymoma (10), with the remaining patients having to continue to take cyclosporine as maintenance therapy (10). In our study, three of six patients continuing to receive cyclosporine obtained complete remission of anemia. In one patient who stopped taking cyclosporine because of complete remission of anemia, relapse of PRCA was recognized. Although the patient started to take cyclosporine again, he became transfusion-dependent, as cyclosporine was not effective. The discontinuation of maintenance cyclosporine therapy led to a relapse of anemia in $86 \%$ of patients with idiopathic PRCA (3). Patients who achieve remission of PRCA associated with thymoma may need to continue to receive cyclosporine, similar to those with idiopathic PRCA. In another study, 2 of 13 patients achieved complete remission with cyclosporine treatment. Although two other patients also achieved transfusion independence on cyclosporine, both relapsed after their doses were decreased due to drug toxicity (11). The response rates of patients with relapse of PRCA were inferior to those of non-treated patients (6). Therapies aside from cyclosporine may be required to overcome PRCA associated with thymoma.

The median overall survival in PRCA associated with thymoma was 142.1 months, and the causes of death were infection, cardiac failure, and thymoma (6). Resistance to immunosuppressive therapies and relapse of anemia were associated with a poor prognosis (6). Maintenance therapies of cyclosporine have been shown to increase the risk of infection, organ failure, and malignancy $(10,11,18)$. In our study, all patients developed pneumonia as a treatmentrelated complication of cyclosporine, and four of six patients died from pneumonia. The treatment for cyclosporinerelated complications may improve the prognosis of PRCA associated with thymoma. Patients receiving immunosuppressive therapies should receive prophylaxis against pneumocystis pneumonia (19). Interestingly, in our study, all patients who received postoperative radiotherapy got pneumonia, and four of these five patients died from pneumonia. Pulmonary fibrosis related to postoperative radiotherapy may influence the occurrence of pneumonia, so such patients should be carefully considered for postoperative radiotherapy. Iron chelation therapy with deferasirox for post-transfusion iron overload improved hematopoiesis in transfusion-dependent patients with aplastic anemia (20), sickle cell disease (21), and PRCA associated with thymoma (22). Iron chelation therapy may improve the outcome of PRCA associated with thymoma in transfusion-dependent patients.

While any conclusions should be tempered due to the limitations associated with the small scale of the present study, we believe that the findings may have a positive impact on the treatment for PRCA associated with thymoma.

\section{Conclusions}

PRCA associated with thymoma was diagnosed postoperatively in three-quarter of patients. We should therefore be alert for the occurrence of PRCA even after resection of thymoma, especially in patients with 


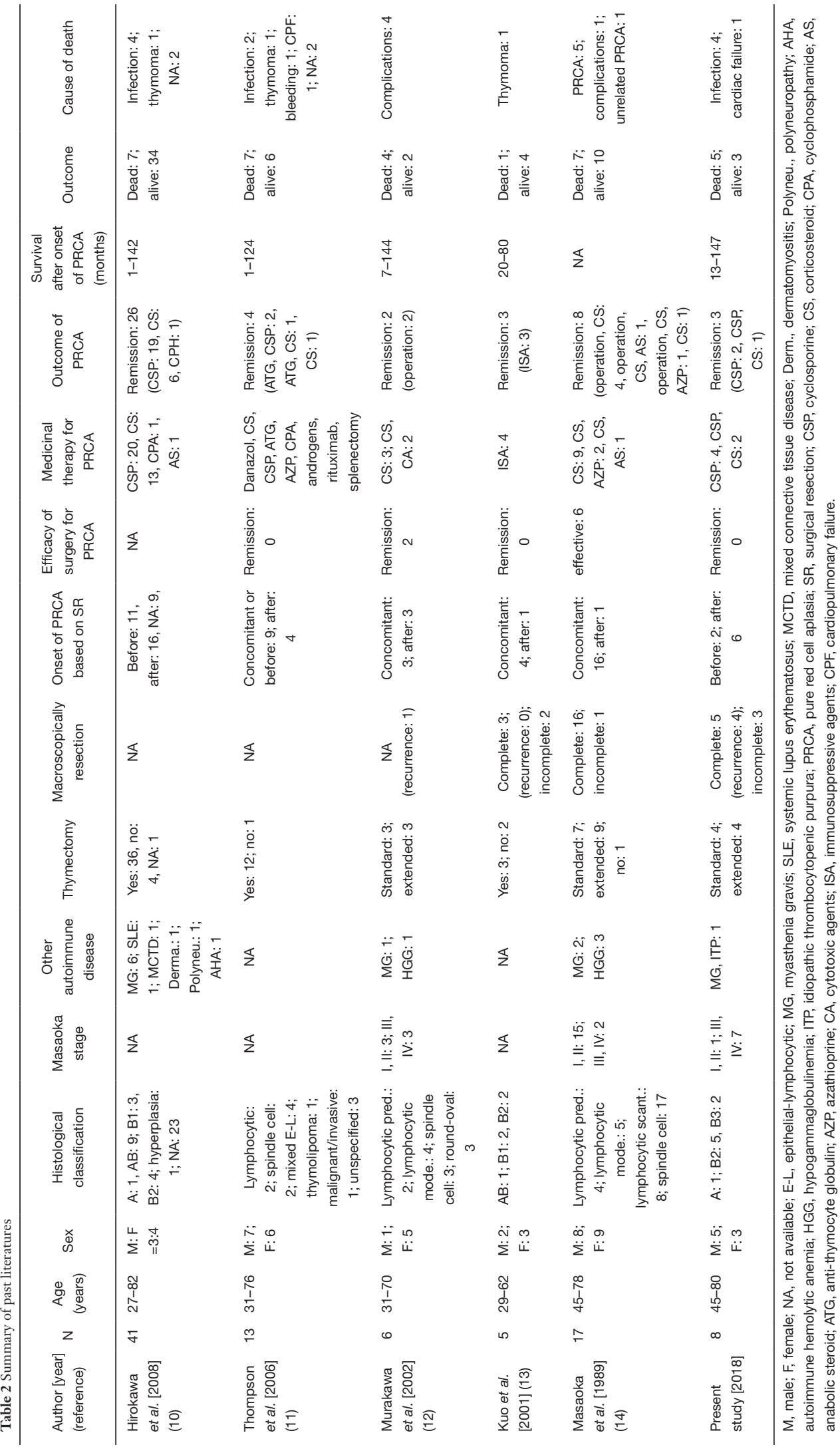


incomplete resection or advanced disease. Cyclosporine was effective against PRCA, but treatment-related complications occurred, particularly pneumonia. As treatment for PRCA associated with thymoma and its complications were combined in a complex manner, treating PRCA associated with thymoma can be quite difficult.

\section{Acknowledgements}

None.

\section{Footnote}

Conflicts of Interest: The authors have no conflicts of interest to declare.

Ethical Statement: This study was approved by the Institutional Review Boards of Nagoya City University Hospital (No. 70-00-0038) and carried out in accordance with the Declaration of Helsinki. All of the patients consented to the use of their tissues for the present analysis.

\section{References}

1. Schmid JR, Kiely JM, Harrison EG Jr, et al. Thymoma associated with pure red-cell agenesis. Review of literature and report of 4 cases. Cancer 1965;18:216-30.

2. Rosenow EC 3rd, Hurley BT. Disorders of the thymus. A review. Arch Intern Med 1984;144:763-70.

3. Sawada K, Hirokawa M, Fujishima N, et al. Long-term outcome of patients with acquired primary idiopathic pure red cell aplasia receiving cyclosporine A. A nationwide cohort study in Japan for the PRCA Collaborative Study Group. Haematologica 2007;92:1021-8.

4. Lacy MQ, Kurtin PJ, Tefferi A. Pure red cell aplasia: association with large granular lymphocyte leukemia and the prognostic value of cytogenetic abnormalities. Blood 1996;87:3000-6.

5. Bernard C, Frih H, Pasquet F, et al. Thymoma associated with autoimmune diseases: 85 cases and literature review. Autoimmun Rev 2016;15:82-92.

6. Hirokawa M, Sawada K, Fujishima N, et al. Long-term outcome of patients with acquired chronic pure red cell aplasia (PRCA) following immunosuppressive therapy: a final report of the nationwide cohort study in 2004/2006 by the Japan PRCA collaborative study group. Br J Haematol 2015;169:879-86.

7. Travis WD, Brambilla E, Burke AP, et al. WHO classification of tumours of the lung, pleura, thymus and heart. Lyon: International Agency for Research on Cancer, 2015:183-211.

8. Masaoka A, Monden Y, Nakahara K, et al. Follow-up study of thymomas with special reference to their clinical stages. Cancer 1981;48:2485-92.

9. Dessypris EN, Lipton JM. Red cell aplasia. In: Greer JP, Foerster J, Lukens JN, et al. Editors. Wintrobe's Clinical Hematology, 11th ed. Philadelphia: Lippincott Williams \& Wilkins, 2004;1421-7.

10. Hirokawa M, Sawada K, Fujishima N, et al. Long-term response and outcome following immunosuppressive therapy in thymoma-associated pure red cell aplasia: a nationwide cohort study in Japan by the PRCA collaborative study group. Haematologica 2008;93:27-33.

11. Thompson CA, Steensma DP. Pure red cell aplasia associated with thymoma: clinical insights from a 50year single-institution experience. Br J Haematol 2006;135:405-7.

12. Murakawa T, Nakajima J, Sato H, et al. Thymoma associated with pure red-cell aplasia: clinical features and prognosis. Asian Cardiovasc Thorac Ann 2002;10:150-4.

13. Kuo T, Shih L. Histologic types of thymoma associated with pure red cell aplasia: a study of five cases including a composite tumor of organoid thymoma associated with an unusual lipofibroadenoma. Int J Surg Pathol 2001;9:29-35.

14. Masaoka A, Hashimoto T, Shibata K, et al. Thymomas associated with pure red cell aplasia. Histologic and followup studies. Cancer 1989;64:1872-8.

15. Zeok JV, Todd EP, Dillon M, et al. The role of thymectomy in red cell aplasia. Ann Thorac Surg 1979;28:257-60.

16. Masuda M, Arai Y, Okumura T, et al. Pure red cell aplasia with thymoma: evidence of T-cell clonal disorder. Am J Hematol 1997;54:324-8.

17. Fujishima N, Hirokawa M, Fujishima M, et al. Oligoclonal $\mathrm{T}$ cell expansion in blood but not in the thymus from a patient with thymoma-associated pure red cell aplasia. Haematologica 2006;91:ECR47.

18. Opelz G, Döhler B. Lymphomas after solid organ transplantation: a collaborative transplant study reports. Am J Transplant 2004;4:222-30.

19. Thomas CF Jr, Limper AH. Pneumocystis pneumonia. $\mathrm{N}$ Engl J Med 2004;350:2487-98.

20. Lee SE, Yahng SA, Cho BS, et al. Improvement in hematopoiesis after iron chelation therapy with deferasirox in patients with aplastic anemia. Acta Haematol 2013;129:72-7. 
21. Vichinsky E, Onyekwere O, Porter J, et al. A randomized comparison of deferasirox versus deferoxamine for the treatment of transfusional iron overload in sickle cell disease. Br J Haematol 2007;136:501-8.

Cite this article as: Moriyama S, Yano M, Haneda H, Okuda K, Kawano O, Sakane T, Oda R, Watanabe T, Nakanishi R. Pure red cell aplasia associated with thymoma: a report of a singlecenter experience. J Thorac Dis 2018;10(8):5066-5072. doi: 10.21037/jtd.2018.07.14
22. Kojima M, Machida S, Sato A, et al. Deferasirox treatment improved hematopoiesis and led to complete remission in a patient with pure red cell aplasia. Int J Hematol 2013;98:719-22. 